\title{
Physio-pathological effects of m6A modification and its potential contribution to melanoma
}

\author{
Y. Liao' ${ }^{1}$ D P. Han ${ }^{1} \cdot$ Y. Zhang ${ }^{2} \cdot$ B. $\mathrm{Ni}^{3}$
}

Received: 4 March 2021 / Accepted: 12 May 2021 / Published online: 8 June 2021

(c) The Author(s) 2021

\begin{abstract}
Methylation of N6-adenosine (m6A) is the most prevalent internal RNA modification and is especially common among the messenger RNAs. These m6A modifications regulate splicing, translocation, stability and translation of RNA through dynamic and reversible interactions with m6A-binding proteins, namely the writers, erasers and readers. RNA methyltransferases catalyze the m6A modifications, while demethylases reverse this methylation. Deregulation of the m6A modification process has been implicated in human carcinogenesis, including melanoma—which carries one of the highest mutant rates. In this review, we provide an up-to-date summary of m6A regulation and its biological impacts on normal and cancer cells, with emphasis on the deregulation of m6A modification and m6A regulators in melanoma. In addition, we highlight the prospective potential of exploiting m6A modification in the treatment of melanoma and non-cancer diseases.
\end{abstract}

Keywords RNA modification $\cdot \mathrm{m6A} \cdot$ Tumor $\cdot$ Melanoma

\section{Introduction}

RNA is a critical carrier of genetic information and catalyzes various biochemical reactions by providing vital information for protein synthesis and acting as a scaffold for attaching the amino acids in proper sequential order. Eukaryotic RNA can be modified in various ways, as previously documented [1]. To date, over 100 kinds of RNA modifications have been identified in eukaryotes and among the various classes of RNAs, including the messenger RNAs (mRNAs) [2, 3], transport RNAs(tRNAs) [4], microRNAs (miRNAs) [5, 6], long non-coding RNAs [3, 7], and circular RNAs [8, 9].

Y. Zhang

zy509419@foxmail.com

$\triangle$ B. Ni

nibing@tmmu.edu.cn

1 Department of Oncology, Chongqing General Hospital, University of Chinese Academy of Sciences, Chongqing 400013, China

2 Chongqing International Institute of Immunology, Chongqing 400018, China

3 Department of Pathophysiology, College of High Altitude Military Medicine, Third Military Medical University, Chongqing 400038, China
Within the various types of RNA modifications, capping at the 5'end and polyA modification at the 3 ' end play very important roles in transcriptional regulation. The methylation modification of mRNA at the 5' cap is a highly regulated process, being crucial for the creation of mature mRNA and involved in maintenance of the mature mRNA's stability, its nuclear exportation, and ability to initiate translation [10]. The polyA modifications, which occur via polyA-binding proteins at the 3' end, similarly contribute to nuclear transport, the maintenance of structural stability, and the functional initiation of translation. Major methylation modifications of RNA include N6-methyladenosine (m6A), 5-methylcytosine, N1-methyladenosine, 5-hydroxymethylcytosine, N6, 2'-O-dimethyladenosine, and 7-methylguanine [11-13]. Of these, m6A modifications mainly occur in the RRACH sequence (where $\mathrm{R}=\mathrm{A}$ or $\mathrm{G}$, and $\mathrm{H}=\mathrm{A}, \mathrm{C}$, or $\mathrm{U}$ ) in nature [14] and it is the most prevalent chemical modification identified for eukaryotic mRNAs [6, 15]. Indeed, it has been estimated that approximately $0.1 \%$ to $0.4 \%$ of adenosines in mRNA are subjected to m6A modification, with, on average, two to three m6A-modified sites per transcript [16].

The m6A modification involves an addition of a methyl group at position N6 of adenosine. Being highly conserved evolutionarily [16], and present in most organisms from bacteria to mammals. m6A is also the most abundant chemical 
modification found on mammalian mRNA and involved in the regulation of a wide array of cellular processes [17]. Not surprisingly, then, alteration of the m6A modification has been found to contribute to the development of various disease states, including obesity [18], infertility [19], autoimmune disease [20], and neurological disease [21]. Deregulation of m6A modification has also recently been implicated in cancer. Although rapid progress has been made in the study of this newly recognized pathogenic mechanism, they are limited by the fact that our knowledge on the regulatory networks of m6Ais not yet complete. In this review, we summarize the most recent findings on the functions of m6A modification in normal and cancer cells, with particular emphasis on the role of m6A modification in melanoma. Finally, we highlight the potential applications and possible future directions in this burgeoning field that will benefit human health.

\section{Basic biology of m6A modification}

Regulation of m6A is mainly accomplished by three homologous factors, namely the so-called "writers", "erasers", and "readers" $[11,22]$. The action of m6A itself is catalyzed by the methyltransferase complex (MTC; i.e. the writer enzymes) and removed by demethylases (i.e. the eraser enzymes). The RNA reader proteins, on the other hand, recognize $\mathrm{m} 6 \mathrm{~A}$, bind to the RNA, and implement its corresponding translation-related functions (Table 1 and Fig. 1).

\section{Methyltransferases/writers}

The M6A writers contribute to the MTC formation and include methyltransferase-like 3 and 14 proteins (METTL3 and METTL14) and their cofactors, the WT1-associated

Table 1 Characteristics and functions of m6A enzymes in RNA metabolism

\begin{tabular}{|c|c|c|c|}
\hline Category & Gene & Characteristics and functions & References \\
\hline \multirow[t]{7}{*}{ Writers } & METTL3 & $\begin{array}{l}\text { Promotes formation of the methyltransferase complex, installs m6A on RNA, and catalyzes m6A } \\
\text { modification }\end{array}$ & {$[14,22,25]$} \\
\hline & METTL14 & $\begin{array}{l}\text { Catalyzes the methylation reaction, mediates protein-protein interaction, and helps METTL3 } \\
\text { recognize the special RNA substrate }\end{array}$ & {$[22,25]$} \\
\hline & METTL16 & $\begin{array}{l}\text { A homologue of METTL3 } \\
\text { Regulator of cellular SAM levels and methylator of U6 small nuclear RNA }\end{array}$ & {$[31,32]$} \\
\hline & WTAP & $\begin{array}{l}\text { Contributes to the localization of METTL3-METTL14 heterodimer to the nuclear speckle } \\
\text { Recruits target RNA for m6A modification } \\
\text { Enhances the catalytic capacity of the writer }\end{array}$ & {$[26]$} \\
\hline & RBM15/15B & Binds the m6A complex and recruit it to special RNA site & {$[27,28]$} \\
\hline & VIRMA & $\begin{array}{l}\text { Recruits the m6A complex to a special RNA site where it interacts with polyadenylation cleavage } \\
\text { factors CPSF5 and CPSF6 }\end{array}$ & {$[30]$} \\
\hline & $\mathrm{ZC} 3 \mathrm{H} 13$ & Bridges WTAP to the mRNA-binding factor to enhance m6A & [29] \\
\hline \multirow[t]{3}{*}{ Erasers } & FTO & Reverses RNA modification and controls cellular homeostasis & {$[33,35]$} \\
\hline & ALKBH5 & $\begin{array}{l}\text { Maintains the balance of m6A levels within the transcriptome } \\
\text { Contributions to in alkylated DNA repair }\end{array}$ & {$[34,35]$} \\
\hline & ALKBH3 & $\begin{array}{l}\text { Performs its demethylation function on tRNA rather than mRNA or rRNA } \\
\text { Generally considered to serve as a DNA repair enzyme }\end{array}$ & {$[37]$} \\
\hline \multirow[t]{12}{*}{ Readers } & YTH family & First five characterized m6A readers & \\
\hline & YTHDC1 & $\begin{array}{l}\text { Contributes to RNA splicing and export } \\
\text { Plays a critical role in the pre-mRNA processing }\end{array}$ & [40] \\
\hline & YTHDC2 & Enhances the translation of target RNA but also reduces the abundance of target RNA & [41] \\
\hline & YTHDF1 & Enhances mRNA translation & [43] \\
\hline & YTHDF2 & Promotes mRNA degradation & [44] \\
\hline & YTHDF3 & Enhances translation and degradation by interacting with YTHDF1 and YTHDF2 & [45] \\
\hline & HNRNP family & Another group of RNA-binding proteins & \\
\hline & HNRNPA2B1 & Mediates miRNA processing by recruiting a microprocessor complex upon binding to the m6A & {$[45,47]$} \\
\hline & HNRNPC/HNRNPG & $\begin{array}{l}\text { Mediate selective splicing of m6A-modified transcripts, but do so through direct interaction whit } \\
\text { m6A-dependent structural switches }\end{array}$ & [45] \\
\hline & eIF3 & Initiates the translation procedure by binding to the m6A site in the 5'-UTR of mRNA & [48] \\
\hline & IGF2BPs & Stabilizes the target gene & [48] \\
\hline & Prrc2a & $\begin{array}{l}\text { Stabilizes mRNA expression by binding to a consensus GGACU motif in the coding sequence in } \\
\text { an m6A-dependent manner }\end{array}$ & [49] \\
\hline
\end{tabular}




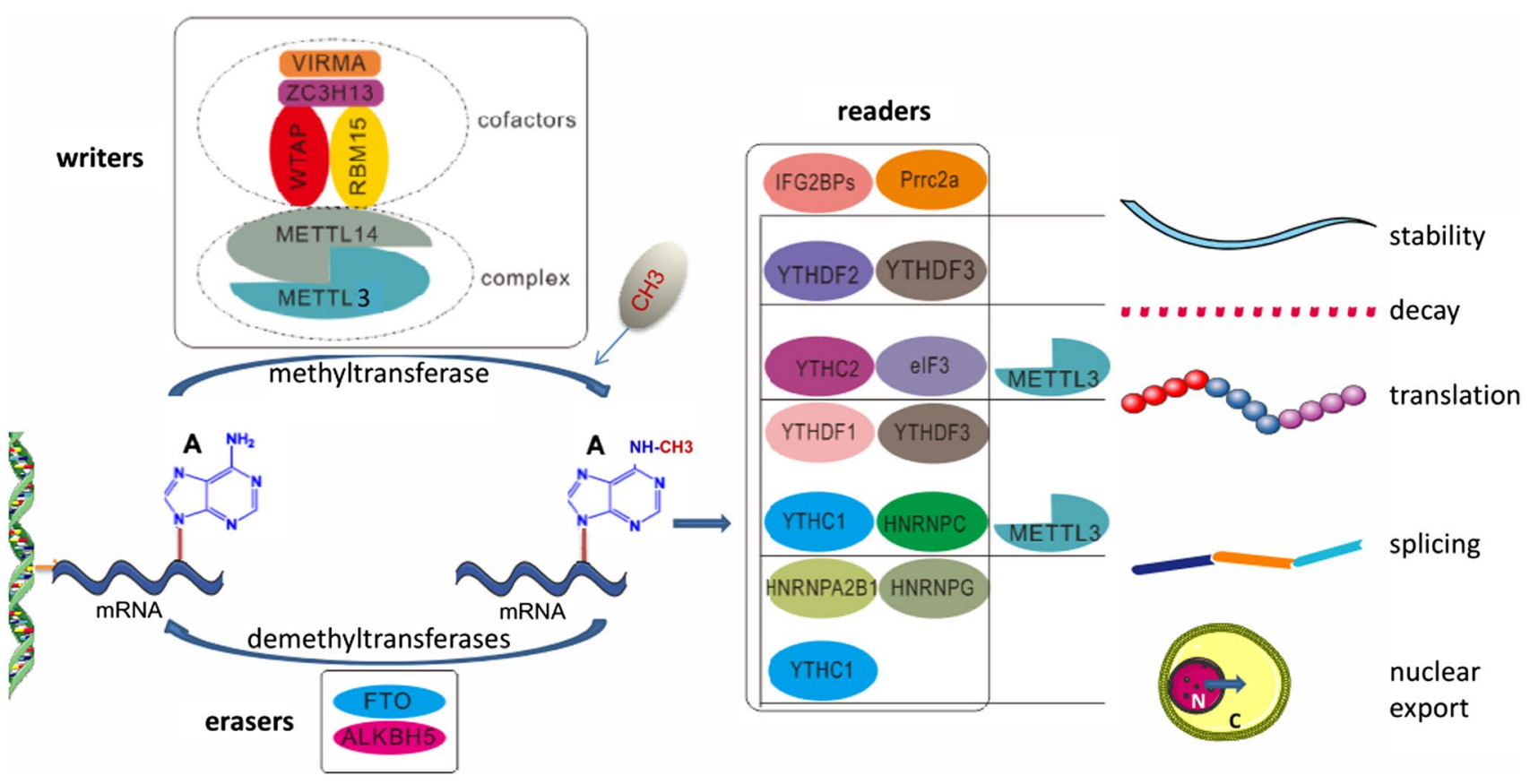

Fig. 1 Schematic process and functions of m6A modification. m6A methylation is catalyzed by the writer complex, which includes METTL3, METTL14, WTAP, VIRMA, RBM15, and ZC3H13. The

m6A modification is removed by the demethylases FTO or ALKBH5. Readers participate in multiple of steps RNA metabolism, including stability, decay, translation, splicing, and nuclear export

protein (WTAP), RNA-binding motif protein 15/15B (RBM15/15B), zinc finger CCCH-type containing 13 (ZC3H13) and Vir-like m6A methyltransferase associated (VIRMA) [11]. METTL3 (also known as MT-A70) was the first component identified in this complex and is the most well understood [23]. It is an S-adenosylmethionine (SAM) binding protein and is highly conserved in various eukaryotic species [24]. It catalyzes the m6A modification and installs m6A on RNA [14, 25]. METTL14 is involved in catalyzing the methylation reaction and mediating protein-protein interaction [22]. In addition, METTL14 supports METTL3 in its recognition of special RNA substrates. WTAP, a splicing factor, is less well understood but studies have indicated a role of mediation in positioning of the heterodimer onto nuclear speckles, as well as for recruitment of the target RNA for m6A modification; the latter would serve to indirectly enhance the catalytic capacity of the writer [26]. Binding of RBM15to the m6A complex facilitates its recruitment to a specific RNA site [27, 28]. ZC3H13 enhances m6A by bridging WTAP to the mRNA-binding factor Nito [29]. VIRMA functions in the 3' untranslated region (UTR) near the stop codon, directing the m6A complex to a special RNA site where it interacts with the polyadenylation cleavage factors CPSF5 and CPSF6 [30].

In addition to the above-described readers that function in complexes, an independently functioning RNA methyltransferase exists. This homologue of METTL3, termed METTL16, has been characterized as a regulator of cellular

SAM levels, a contributor to pre-RNA splicing, and a methylator of U6 small nuclear RNA [31, 32].

\section{Demethylases/erasers}

The dynamic and reversible m6A process also relies on demethylases (erasers). Fat mass and obesity-associated protein (FTO) was the first demethylase discovered, and its ability to reverse RNA modification and control cellular homeostasis is now well characterized. FTO has also been implicated in the adipogenic process, exerting its activity through modulation of the alternative splicing of RUNX1T1 [33]. The AlkB homolog 5 RNA demethylaseALKBH5, expressed primarily in the testes, was the second demethylase identified [34]. The distinct physiological functions of ALKBH5 include regulatory contributions to alkylated DNA repair and maintaining the balance of m6A levels within the transcriptome- the latter being accomplished by working in tandem with FTO [35]. The most recently discovered demethylase, ALKBH3, appears to preferentially demethylate tRNA rather than mRNA or rRNA [36] and is generally considered to serve as a DNA repair enzyme [37].

\section{Readers}

The readers are required for the m6A-modified RNA's capacity to perform its various biological functions in mRNA translation, degradation, splicing, export, and folding 
[38]. The first five m6A readers characterized in human represented the YT521-B homology (YTH) domain family [2]. These five proteins, which include two YTHDC members and three YTHDF members, share a conserved m6A-binding domain. YTHDC1 is a nuclear m6A reader, showing almost completely overlapping sites with m6A in nuclear RNAs. Functionally, YTHDC1 contributes to RNA splicing and export, and plays a critical role in the pre-mRNA processing in the oocyte nucleus via interaction with the pre-mRNA3'end processing factors CPSF6, SRSF3, and SRSF7 [39, 40]. YTHDC2 serves to increase the translation efficiency of target RNA but to also reduce the abundance of target RNA [41]. The three YTHDF proteins recognize the consensus sequence G[G $>A$ Am6ACU [42]. YTHDF1 evokes m6Acontaining mRNA translation, upon interaction with an array of translation initiation factors [2, 43]. YTHDF2 harbors a $\mathrm{P} / \mathrm{Q} / \mathrm{N}$ motif with high affinity for mRNAs, resulting in their localization in processing bodies (so-called "P bodies"); the accumulated mRNA undergoes turnover by RNA degradation, a process which involves the YTHDF2 N-terminus and the deadenylase complex CCR4-NOT in mammalian cells [44]. Finally, YTHDF3 enhances RNA translation by interacting with YTHDF1and promotes RNA degradation by associating with YTHDF2 [45].

The heterogeneous nuclear ribonucleoprotein (HNRNP) family of RNA-binding proteins shares a low-complexity domain at the C-terminus. The HNRNPA2B1 member mediates miRNA processing by recruiting a microprocessor complex upon binding to the m6A RGAC motif that is present in a subset of primary miRNA transcripts; this process ultimately facilitates alternative splicing, in a manner similar to that of METTL3 [46, 47]. The HNRNPC and HNRNPG members mediate selective splicing of m6A-modified transcripts, but do so through direct interaction with m6Adependent structural switches [36]. The translation process itself is then initiated upon the translation initiation factor 3 binding to the m6A site in the 5'-UTR of mRNA, working in conjunction with the insulin-like growth factor $2 \mathrm{mRNA}$ binding protein family members IGF2BP $1 / 2 / 3$ stabilizingthe target gene [48]. The mRNA expression process is further stabilized by interaction between another m6A reader protein, the proline-rich coiled coil 2 A (Prrc2a), which binds to a consensus GGACU motif in the coding sequence [49].

Although each component involved in m6A modification has its own characteristics and functions, they do not function in a mutually exclusive manner. There is much evidence demonstrating collaboration between the writers, erasers, and readers, particularly in the context of cancer pathophysiology [50, 51]. For instance, expression of the YTHDC1 reader shows significant correlation with the expression of several writers, including METTL3 and METTL14; moreover, these correlations are even higher between genes expressing proteins in the same functional complexes, such as RBM15 and WTAP, of the human spliceosome. As these writers, erasers, and readers are known to interact with each other frequently in protein-protein interaction networks, it follows that cross-talk among the enzymes of RNA methylation plays critical roles in the development and progression of various diseases [38].

\section{Pathophysiological roles of $\mathrm{m6A}$ modification in tumor diseases}

m6A modification contributes to an array of processes in the formation and progression of cancer, and it can modulate the biological behavior of cancer cells in a variety of ways [52], from cancer stem cell formation and the epithelial-mesenchymal transition to signal transduction and tumor metabolism [17]. Often these, changes occur because of writers adding m6A modifications in the mRNA of oncogenes or tumor suppressor genes and readers that then recognize these biomarkers. Readers can affect the expression of both oncogenes (upregulation) and tumor suppressor genes (downregulation). Conversely, modification removal of the m6A modification by erasers negates the readers' activities, resulting in the opposite gene regulation effects (oncogene upregulation and tumor suppressor gene downregulation) [53].

Although aberrant m6A modification more commonly contributes to tumorigenesis and tumor progression, recent studies have revealed that abnormal m6A levels can also cause tumor suppression [9]. In glioblastoma stem cells (GSCs), METTL3 promotes mRNA methylation, enhances the stability and expression of SRY-box transcription factor 2 (SOX2), and promotes maintenance and radio-resistance [54]. Additionally, METTL3 has been shown to alter A-to-I and C-to-U RNA-editing events by differentially regulating the RNA-editing enzymes adenosine deaminase acting on RNA and apolipoprotein B mRNA-editing enzyme catalytic subunit $3 \mathrm{~A}$. In contrast, some studies have shown that METTL3 is beneficial in the prevention of GSC self-renewal and tumorigenicity [55]. Thus, METTL3 is characterized as a crucial factor throughout the many steps of RNA processing, thereby coordinating the oncogenic pathway in GSCs [56]. In colorectal carcinoma, in particular, METTL3 can facilitate carcinogenesis via an IGF2BP2-dependent process that inhibits SOX2 degradation [57]. In contrast, however, METTL3 interaction with the p38/ERK pathway inhibits the proliferative, migratory, and invasive capacities of colorectal carcinoma cells [58]. In hepatocellular carcinoma (HCC), METTL3-mediated increase in the m6A level of SOCS2 mRNA promotes degradation of this ubiquitous cytokine signaling transducer through an m6A YTHDF2-dependent mechanism; the decreased expression of SOCS2 promotes HCC progression [50]. Simultaneously, YTHDF2 exerts an 
inhibitory effect on HCC, inhibiting growth of tumor cells and vessels by processing interleukin 11 (IL11) mRNA and serpin family E member 2 (SERPINE2) mRNA [59].

A single m6A modification factor can perform in different ways in different tumors. For example, in lung cancer, YTHDF2 promotes the tumor cell growth by binding directly to the m6A-modified site in the 6-phosphogluconate dehydrogenase (6PGD) 3'-UTR. The resultant increases in translation of the 6PGD mRNA and flux through the pentose phosphate pathway promote the tumor growth [60]. In acute myeloid leukemia, overexpressed YTHDF2 reduces the half-life of tumor necrosis factor receptor superfamily member 2, as well as other m6A-modified transcripts, supporting the leukemic stem cell niche [61]. In breast cancer, a YTHDF2-dependent mechanism underlies the degradation of the BCL2 interacting protein 3 (BNIP3) mRNA, which would otherwise encode an apoptosis-promoting protein that is a downstream target of FTO-mediated m6A modifications [62]. YTHDF2 can also inhibit HCC tumor cells and related vessels by processing IL11 and SERPINE2 mRNAs [59].

\section{Therapeutic applications}

A growing number of studies are revealing many potential therapeutic applications of m6A for clinical use. For example, (i) tumor neoantigens generate spontaneous antitumor immunity and can serve as biomarkers for evaluating clinical responses to immunotherapies [63]. A recent study indicated that YTHDF1 can suppress the cross-priming ability of dendritic cells. Loss of YTHDF1 then promotes infiltration of neo-antigen-specific $\mathrm{CD} 8^{+} \mathrm{T}$ cells and enhances the crosspresentation of tumor antigens to inhibit tumors growth. Furthermore, given that YTHDF1 depletion promotes interferon gamma (IFN- $\gamma$ ) production, followed by an increase in PD-L1 in $\mathrm{CD}^{+} \mathrm{T}$ cells [64], it follows that combining a PD-L1 checkpoint inhibitor with YTHDF1 depletion would produce a stronger therapeutic efficacy. In combination with the more recent checkpoint blockade strategy, YTHDF1 could be a potential new therapeutic target for immunotherapy. (ii) FTO, which had previously only been studied as an inhibitor in tumors, was found to be higher in cervical squamous cell carcinoma tissue than in the corresponding paracancerous tissues. FTO increases the expression of excision repair cross-complementation group, thereby contributing to chemo- and radio-resistance and showing potential clinical significance for the treatment of this cancer type [65, 66]. (iii) In a mouse model of leukemia, cells with mRNA m6A hypo-methylation and FTO upregulation was found to promote tolerance to tyrosine kinase inhibitor treatment and growth rates. FTO deactivation (m6A methylation inducement by genetic or pharmacologic means) restores the sensitivity to tyrosine kinase inhibitors [66]. (iv) Chen et al. [67] showed that m6A RNA methylation regulators are closely related to malignant clinico-pathological features of breast cancer and identified a prognostic bio-signature, composed of the FTO, YTHDC1 and WTAP m6A RNA methylation regulators. We speculate that similar studies will highlight the therapeutic applicability of different m6A RNA methylation regulators to other tumors [68].

\section{m6A involvement in melanoma}

Melanoma is the fifth-most common cause of cancer overall in the United States [69]. Between 2006 and 2015, the overall incidence rate of melanoma increased from 200.1 to 229.1 cases per million person-years and the death rate is more than 9000 per year [70]. Although progress has been made in the treatment of advanced melanoma [71, 72], there remain no proper curative treatments currently available. Melanoma is a type of epithelial malignant tumor that originates from melanocytes [73]. Advances in sequencing technology have led to the identification of pathogenic mutations in the B-Raf proto-oncogene (BRAF) and related signaling pathways, such as the mitogen-activated protein kinase (MAPK) pathway and phosphatidylinositol 3-kinaseAKT pathway $[74,75]$. The BRAF mutations are associated with decreased disease-free and melanoma-specific survival. These discoveries have led to the emergence of targeted drug treatments for melanoma, such as BRAF inhibitors, MAP2K7 (also known as MEK), and MAPK1 (also known as ERK) [76, 77]. The Telomerase Reverse Transcriptase (hTERT) promoter mutations, which were first identified in melanomas, often in combination with BRAF mutations, were reported to activate the telomerase and to be implicated in tumorigenesis $[78,79]$. Also, some studies linked these mutations to the treatment efficacy or resistance [79, 80]. However, the actual pathological mechanism of melanoma remains unknown. Considering that $\mathrm{m} 6 \mathrm{~A}$ methylation is closely related to tumorigenesis and tumor development [65, 81], several studies have investigated the roles for such in the pathological processes of melanoma (Fig. 2).

Though many cancers show deregulated expression of m6A and METTL3, the role of each in melanoma still remains to be fully elucidated. Using in vitro systems, Dahal et al. [82] found that melanoma cell lines express higher levels of METTL3 than normal melanocytes. Silencing of the METTL3 gene expression reduced not only the m6A activity but also the cells' capacities for colony formation and invasiveness, with over-expression of the METTL3 gene producing the opposite effects upon accumulation of MMP2 and $\mathrm{N}$-cadherin. Overexpression of m6A catalytic site mutant in METTL3 did not produce a similar increase in MMP2, suggesting that m6A activity of METTL3 is important for melanoma cell invasiveness [83]. Though the results need 
Fig. 2 Potential roles of m6A modification in melanoma pathogenesis. The effect of m6A on melanoma cells is reflected in the regulation of cancerrelated gene expression

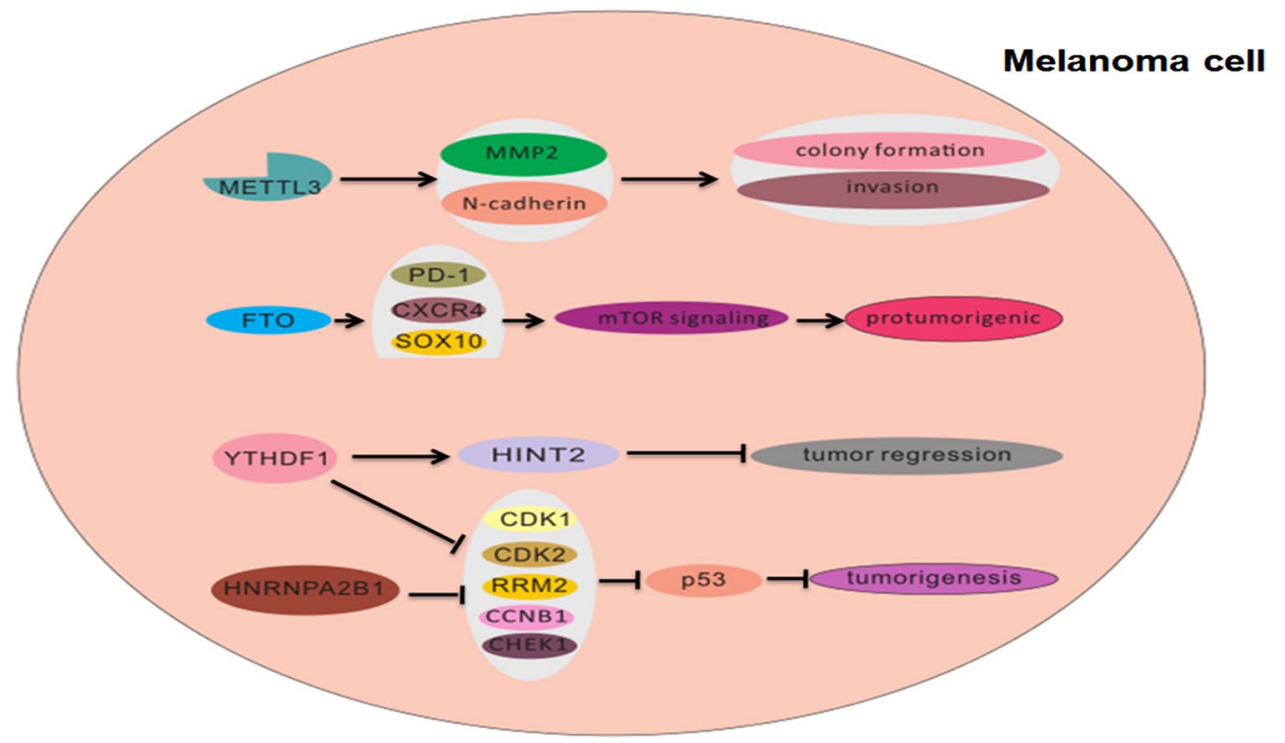

to be confirmed by in vivo studies, they suggest a potential therapeutic benefit of METTL3 inhibitors in melanoma.

Research on FTO has suggested that m6A RNA methylation is reversible and dynamic and may have crucial physiological and pathological functions [84]. Human melanoma tissues show upregulated FTO [85]. FTO expression is regulated by autophagy pathway and nuclear factor kappa B pathways and can be induced by metabolic stress; the FTO protein itself regulates the expression of known melanoma-related genes encoding PD-1 (PDCD1), CXCR4, and SOX10. Indeed, knockdown of FTO reduced the levels of these proteins but also had a negative impact on the phosphorylation of p70s6K, a substrate of mTOR activation [85]. PD-1 activates pathways downstream of mTOR, itself a tumorigenic factor that promotes melanoma tumor growth [86]. Increasing the general knowledge on the regulatory mechanisms of PD-1 under normal physiologic conditions may help us to better understand its regulatory roles in cancer biology and immunotherapy response in melanoma; indeed, one study has already used PD-1 to successfully target immune cells as a cancer immunotherapy [87]. The collective results from these PD-1 studies have indicated the critical role of melanoma cell-intrinsic FTO in promoting melanoma resistance, such as to anti-PD-1 blockade [85] (Fig. 3). Ultimately, however, they suggest that the regulation of these melanoma-promoting genes by FTO and other proteins might critically affect gene expression in melanoma (e.g., the methyltransferases METTL3 and METTL14) and further studies on the therapeutic potential of such are warranted.

As described above, the regulation of gene expression by $\mathrm{m} 6 \mathrm{~A}$ is executed mostly through the readers, including YTH domain-containing family proteins in mammalian cells. Yang and colleagues [85] found that knocking down only YTHDF2 significantly increased the mRNA stability of PD-1 (PDCD1), CXCR4, and SOX10; in conjunction, the proliferative and migratory capacities of the melanoma cells were increased (in vitro) as was tumor growth (in vivo). In melanoma cells, YTHDF2 would serve as a tumor suppressor, mediating the downregulation of the FTO target genes. Although in normal melanoma, the other family member YTHDF1 does not reduce cell proliferation and migration, it can promote translation of the histidine triad nucleotidebinding protein 2 tumor suppressor in ocular melanoma, being shown to promote tumor regression in vitro and in vivo [88].

One systematic analysis revealed that the expressions of YTHDF1 and HNRNPA2B1 were upregulated in melanoma [89]. Expression screening for both of these genes, in combination, was also found to improve melanoma diagnosis rates by about $10 \%$ (versus mono-gene screening). Furthermore, the study found that genes related to p53-signaling (namely, CDK2, CDK1, RRM2, CCNB1, and CHEK1) positively correlated with the observed YTHDF1 or HNRNPA2B1 upregulation. Thus, both genes may affect m6A modification of the identified five genes in particular, promoting the ability of the encoded proteins to inhibit p53 and suppress tumorigenesis. Finally, the study found that upregulating mutations in YTHDF1 and HNRNPA2B1 correlated with tumor stage and treatment response in patients, further supporting their roles, asm6A regulatory genes, in melanoma.

m6A modification contributes to an array of processes in the formation and progression of cancer. In malignant ocular melanoma, the "writer" METTL3, lower expression predicted earlier recurrence and enhanced aggressiveness, significantly decreased in m6A modification in cellular mRNAs, while the "eraser" ALKBH5, the expression indicated a poor prognosis, was observed the opposite trend. 
Fig. 3 Mechanistic illustrations of the regulatory and functional roles for FTO in melanoma. FTO can regulate cell-intrinsic expressions of PD-1, CXCR4, and SOX10 to promote the growth and anti-PD-1 response of melanoma

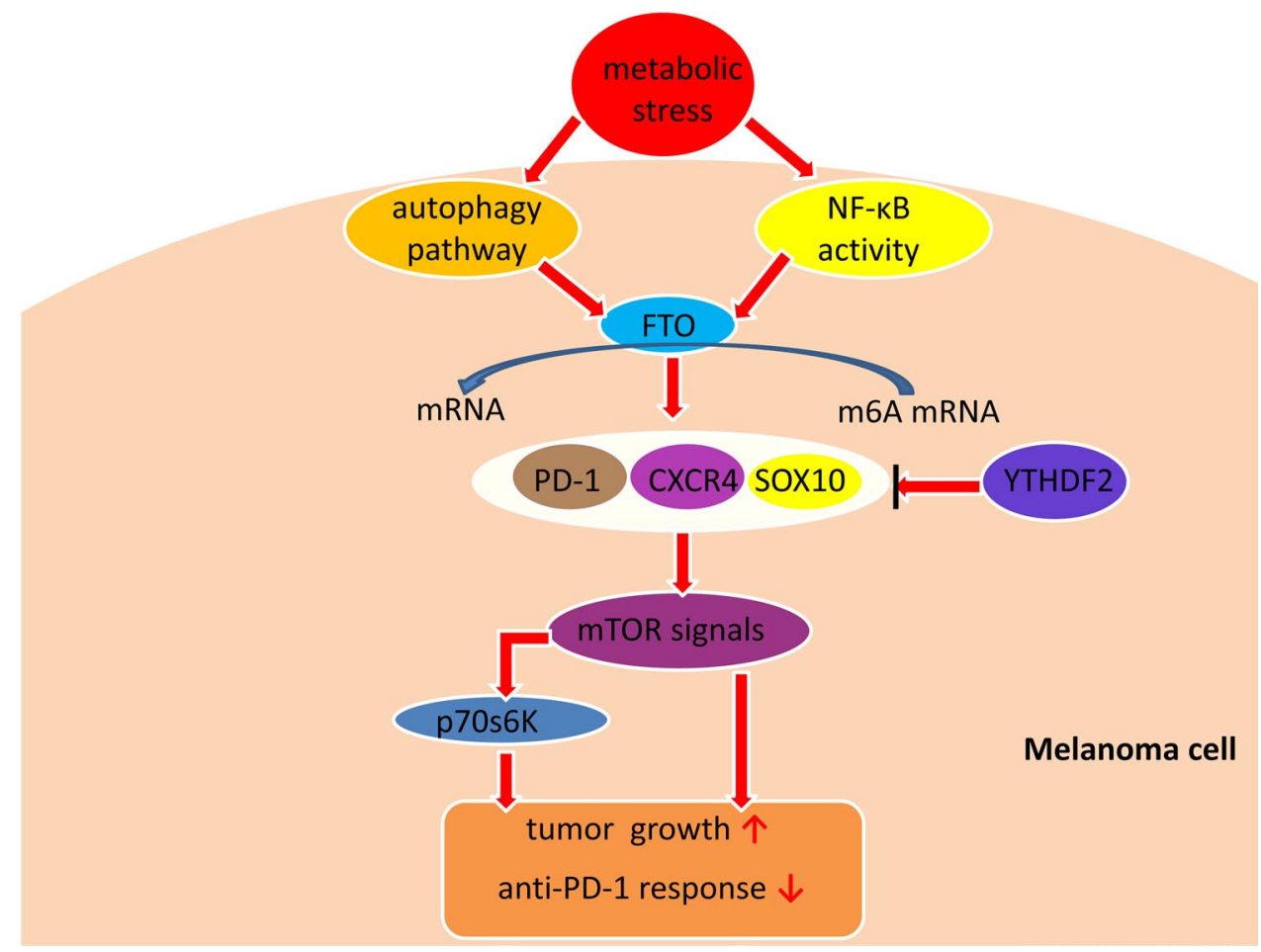

This study indicates that the disturbance of global m6A homeostasis, which depends on the balance of "writers" and "erasers" of m6A modification, is a key driver in the regulation of tumor formation [88].

In short, these studies on m6A in melanoma have advanced our understanding of the pathogenesis and biological characteristics of melanoma, in general, and may provide foundational data to improve future diagnosis and development of molecular targeted therapy and immunotherapies. However, research on the role of m6a in melanoma is relatively limited, additional research is necessary to elucidate the molecular mechanisms of m6A in melanoma.

\section{Conclusion and perspectives}

The m6A modification of RNA is becoming recognized as an important post-transcriptional regulation of gene expression, both under physiologic and pathogenic conditions. Deregulation of the underlying m6A regulators affects a vast array of downstream targets, disrupting mRNA stability and impeding translation efficiency. The implications of m6A modification in human carcinogenesis have been demonstrated in different cancer types, including melanoma. However, the mechanisms underlying m6A modification and $\mathrm{m} 6 \mathrm{~A}$ regulators are heterogeneous and complex. Identification of cancer-specific m6A modifications will be important, as they may serve as potential diagnostic and prognostic markers or as targets for development of novel therapies.
While the effects of m6A modification are known for many types of tumors, effects on non-tumor diseases and physiological processes are only beginning to be recognized. Many of the m6A transcripts encoded by viruses have been identified [90] and their functions in viral-host interactions are believed to be important in human disease. For example, m6A modifications to RNAs of the human respiratory syncytial virus (RSV) enhance viral replication and pathogenesis; results from knockdown studies of the m6A methyltransferases and demethylases have suggested that viral m6A methylation could be a therapeutic target [91]. In addition, under stress conditions, mRNA methylation in human and mouse cardiomyocytes is highly dynamic; the mRNA methylome may be a manipulable regulator of translational efficiency, based upon its effects on transcript stability [92]. Once more m6A methylation mechanisms are elucidated, targeted molecular manipulation could be a powerful approach to preventive care, such as worsening of cardiac stress.

Interestingly, m6A modification has also been implicated in homeostasis of neuronal and immune systems. Chen and colleagues [93] found that depletion of METTL3, in particular, significantly reduced m6A levels in adult neural stem cells (aNSCs) and inhibited their proliferation. Ultimately, neuronal development was inhibited and differentiation of the aNSCs became skewed towards a glial lineage, effects that were rescued by Ezh2 over-expression [93]. These results may provide insight into central nervous system diseases. M6A modification was also recently characterized as 
a crucial regulator of $\mathrm{T}$ cell homeostasis and the immune response to bacterial or viral infection [31], highlighting an immunotherapeutic potential for such.

So far, m6A modification has been found to play significant roles in cancers and it can provide a series of new pharmacological targets. Taketo $\mathrm{K}$ et al. show that the METTL3-depleted have higher sensitivity to anti-cancer reagents, such as gemcitabine, 5-fluorouracil, cisplatin and irradiation, than those without METTL3-depleted in pancreatic cancer patients [94]. Whether the same results will apply to melanoma remains unknown, thus further studies should be performed to understand m6 A modification in melanoma and other cancers and more highly selective and potent inhibitors of m6A modification should be required. It is well known that immunotherapy plays an important role in the treatment of melanoma, a variety of researches have confirmed RNA m6A methylation can modulate innate/adaptive immunity responses [95]. For instance, $\mathrm{T}$ cells modified to carry $\mathrm{m}^{6} \mathrm{~A}$-modifying agents could be an efficient therapeutic strategy for various autoimmune diseases [96], which also suggests the potential application of such strategy in cancer immunotherapy. Although these results have not yet been verified in vivo and in clinical trials, it can provide potential pharmacological targets for anti-cancer drug development and cancer immunotherapy for melanoma.

Funding This work was supported by grants from the National Key Research and Development Project (No. 2016YFA0502203). The funders had no role in the study design, data analysis, or decision to publish.

\section{Declarations}

Conflict of interests The authors declare no conflicts of interest related to this publication.

Ethical approval This article does not contain any studies with human participants performed by any of the authors.

Informed consent For this type of study, formal constent is not required.

Open Access This article is licensed under a Creative Commons Attribution 4.0 International License, which permits use, sharing, adaptation, distribution and reproduction in any medium or format, as long as you give appropriate credit to the original author(s) and the source, provide a link to the Creative Commons licence, and indicate if changes were made. The images or other third party material in this article are included in the article's Creative Commons licence, unless indicated otherwise in a credit line to the material. If material is not included in the article's Creative Commons licence and your intended use is not permitted by statutory regulation or exceeds the permitted use, you will need to obtain permission directly from the copyright holder. To view a copy of this licence, visit http://creativecommons.org/licenses/by/4.0/.

\section{References}

1. Uddin MB, Wang Z, Yang C. Dysregulations of functional RNA modifications in cancer, cancer stemness and cancer therapeutics. Theranostics. 2020;10(7):3164-89.

2. Wang X, Zhao BS, Roundtree IA, Lu Z, Han D, Ma H, Weng X, Chen K, Shi H, He C. N(6)-methyladenosine modulates messenger RNA translation efficiency. Cell. 2015;161(6):1388-99.

3. Pan T. N6-methyl-adenosine modification in messenger and long non-coding RNA. Trends Biochem Sci. 2013;38(4):204-9.

4. Wei J, Liu F, Lu Z, Fei Q, Ai Y, He PC, Shi H, Cui X, Su R, Klungland A, et al. Differential m(6)A, m(6)Am, and m(1)A demethylation mediated by FTO in the cell nucleus and cytoplasm. Mol Cell. 2018;71(6):973-985 e975.

5. Lewis BP, Burge CB, Bartel DP. Conserved seed pairing, often flanked by adenosines, indicates that thousands of human genes are microRNA targets. Cell. 2005;120(1):15-20.

6. Meyer KD, Saletore Y, Zumbo P, Elemento O, Mason CE, Jaffrey SR. Comprehensive analysis of mRNA methylation reveals enrichment in 3' UTRs and near stop codons. Cell. 2012;149(7):1635-46.

7. Linder B, Grozhik AV, Olarerin-George AO, Meydan C, Mason CE, Jaffrey SR. Single-nucleotide-resolution mapping of m6A and m6Am throughout the transcriptome. Nat Methods. 2015;12(8):767-72.

8. Wei W, Ji X, Guo X, Ji S. Regulatory Role of N(6) -methyladenosine $(\mathrm{m}(6)$ A) methylation in RNA processing and human diseases. J Cell Biochem. 2017;118(9):2534-43.

9. Zhang L, Hou C, Chen C, Guo Y, Yuan W, Yin D, Liu J, Sun Z. The role of $\mathrm{N}(6)$-methyladenosine $(\mathrm{m}(6) \mathrm{A})$ modification in the regulation of circRNAs. Mol Cancer. 2020;19(1):105.

10. Cowling VH. Regulation of mRNA cap methylation. Biochem J. 2009;425(2):295-302.

11. Li J, Yang X, Qi Z, Sang Y, Liu Y, Xu B, Liu W, Xu Z, Deng Y. The role of mRNA m(6)A methylation in the nervous system. Cell Biosci. 2019;9:66.

12. Wei CM, Gershowitz A, Moss B. Methylated nucleotides block 5' terminus of HeLa cell messenger RNA. Cell. 1975;4(4):379-86.

13. Dubin DT, Taylor RH. The methylation state of poly A-containing messenger RNA from cultured hamster cells. Nucleic Acids Res. 1975;2(10):1653-68.

14. Bokar JA, Shambaugh ME, Polayes D, Matera AG, Rottman FM. Purification and cDNA cloning of the AdoMet-binding subunit of the human mRNA (N6-adenosine)-methyltransferase. RNA. 1997;3(11):1233-47.

15. Wang X, Wu R, Liu Y, Zhao Y, Bi Z, Yao Y, Liu Q, Shi H, Wang F, Wang Y. m(6)A mRNA methylation controls autophagy and adipogenesis by targeting Atg5 and Atg7. Autophagy. 2020;16(7):1221-35.

16. Fu Y, Dominissini D, Rechavi G, He C. Gene expression regulation mediated through reversible m(6)A RNA methylation. Nat Rev Genet. 2014;15(5):293-306.

17. Chen M, Wong CM. The emerging roles of N6-methyladenosine (m6A) deregulation in liver carcinogenesis. Mol Cancer. 2020;19(1):44.

18. Chen J, Du B. Novel positioning from obesity to cancer: FTO, an m(6)A RNA demethylase, regulates tumour progression. J Cancer Res Clin Oncol. 2019;145(1):19-29.

19. Ivanova I, Much C, Di Giacomo M, Azzi C, Morgan M, Moreira PN, Monahan J, Carrieri C, Enright AJ, O'Carroll D. The RNA $\mathrm{m}(6) A$ reader YTHDF2 is essential for the post-transcriptional regulation of the maternal transcriptome and oocyte competence. Mol Cell. 2017;67(6):1059-1067e1054.

20. Winkler R, Gillis E, Lasman L, Safra M, Geula S, Soyris C, Nachshon A, Tai-Schmiedel J, Friedman N, Le-Trilling VTK, 
et al. m(6)A modification controls the innate immune response to infection by targeting type I interferons. Nat Immunol. 2019;20(2):173-82.

21. Weng YL, Wang X, An R, Cassin J, Vissers C, Liu Y, Liu Y, Xu $\mathrm{T}$, Wang $\mathrm{X}$, Wong SZH, et al. Epitranscriptomic m(6)A regulation of axon regeneration in the adult mammalian nervous system. Neuron. 2018;97(2):313-325e316.

22. Lee M, Kim B, Kim VN. Emerging roles of RNA modification: $\mathrm{m}(6) \mathrm{A}$ and U-tail. Cell. 2014;158(5):980-7.

23. Liu J, Yue Y, Han D, Wang X, Fu Y, Zhang L, Jia G, Yu M, Lu Z, Deng $X$, et al. A METTL3-METTL14 complex mediates mammalian nuclear RNA N6-adenosine methylation. Nat Chem Biol. 2014;10(2):93-5.

24. Li F, Yi Y, Miao Y, Long W, Long T, Chen S, Cheng W, Zou $\mathrm{C}$, Zheng Y, Wu X, et al. N(6)-methyladenosine modulates nonsense-mediated mRNA decay in human glioblastoma. Cancer Res. 2019;79(22):5785-98.

25. Wang P, Doxtader KA, Nam Y. Structural basis for cooperative function of Mettl3 and Mettl14 methyltransferases. Mol Cell. 2016;63(2):306-17.

26. Ping XL, Sun BF, Wang L, Xiao W, Yang X, Wang WJ, Adhikari $\mathrm{S}$, Shi Y, Lv Y, Chen YS, et al. Mammalian WTAP is a regulatory subunit of the RNA N6-methyladenosine methyltransferase. Cell Res. 2014;24(2):177-89.

27. Patil DP, Chen CK, Pickering BF, Chow A, Jackson C, Guttman M, Jaffrey SR. m(6)A RNA methylation promotes XIST-mediated transcriptional repression. Nature. 2016;537(7620):369-73.

28. Coker H, Wei G, Moindrot B, Mohammed S, Nesterova T, Brockdorff $\mathrm{N}$. The role of the Xist 5' m6A region and RBM15 in X chromosome inactivation. Wellcome Open Res. 2020;5:31.

29. Wen J, Lv R, Ma H, Shen H, He C, Wang J, Jiao F, Liu H, Yang $\mathrm{P}$, Tan $\mathrm{L}$, et al. Zc3h13 regulates nuclear RNA m(6)A methylation and mouse embryonic stem cell self-renewal. Mol Cell. 2018;69(6):1028-1038e1026.

30. Yue Y, Liu J, Cui X, Cao J, Luo G, Zhang Z, Cheng T, Gao M, Shu X, Ma H, et al. VIRMA mediates preferential m(6)A mRNA methylation in 3'UTR and near stop codon and associates with alternative polyadenylation. Cell Discov. 2018;4:10.

31. Zhang $\mathrm{C}, \mathrm{Fu} \mathrm{J}$, Zhou Y. A review in research progress concerning m6A methylation and immunoregulation. Front Immunol. 2019;10:922

32. Warda AS, Kretschmer J, Hackert P, Lenz C, Urlaub H, Hobartner C, Sloan KE, Bohnsack MT. Human METTL16 is a N(6)-methyladenosine $(\mathrm{m}(6) \mathrm{A})$ methyltransferase that targets pre-mRNAs and various non-coding RNAs. EMBO Rep. 2017;18(11):2004-14.

33. Merkestein M, Laber S, McMurray F, Andrew D, Sachse G, Sanderson J, Li M, Usher S, Sellayah D, Ashcroft FM, et al. FTO influences adipogenesis by regulating mitotic clonal expansion. Nat Commun. 2015;6:6792.

34. Zheng G, Dahl JA, Niu Y, Fedorcsak P, Huang CM, Li CJ, Vagbo $\mathrm{CB}$, Shi Y, Wang WL, Song SH, et al. ALKBH5 is a mammalian RNA demethylase that impacts RNA metabolism and mouse fertility. Mol Cell. 2013;49(1):18-29.

35. Huang Y, Yan J, Li Q, Li J, Gong S, Zhou H, Gan J, Jiang H, Jia GF, Luo C, et al. Meclofenamic acid selectively inhibits FTO demethylation of m6A over ALKBH5. Nucleic Acids Res. 2015;43(1):373-84.

36. Ueda Y, Ooshio I, Fusamae Y, Kitae K, Kawaguchi M, Jingushi K, Hase H, Harada K, Hirata K, Tsujikawa K. AlkB homolog 3-mediated tRNA demethylation promotes protein synthesis in cancer cells. Sci Rep. 2017;7:42271.

37. Beharry AA, Lacoste S, O'Connor TR, Kool ET. Fluorescence monitoring of the oxidative repair of DNA alkylation damage by ALKBH3, a prostate cancer marker. J Am Chem Soc. 2016;138(11):3647-50.
38. Liu Q, Gregory RI. RNAmod: an integrated system for the annotation of mRNA modifications. Nucleic Acids Res. 2019;47(W1):W548-55.

39. Kasowitz SD, Ma J, Anderson SJ, Leu NA, Xu Y, Gregory BD, Schultz RM, Wang PJ. Nuclear m6A reader YTHDC1 regulates alternative polyadenylation and splicing during mouse oocyte development. PLoS Genet. 2018;14(5):e1007412.

40. Roundtree IA, Luo GZ, Zhang Z, Wang X, Zhou T, Cui Y, Sha J, Huang X, Guerrero L, Xie P, et al. YTHDC1 mediates nuclear export of N(6)-methyladenosine methylated mRNAs. Elife. 2017;6:e31311.

41. Hsu PJ, Zhu Y, Ma H, Guo Y, Shi X, Liu Y, Qi M, Lu Z, Shi $\mathrm{H}$, Wang $\mathrm{J}$, et al. Ythdc2 is an $\mathrm{N}(6)$-methyladenosine binding protein that regulates mammalian spermatogenesis. Cell Res. 2017;27(9):1115-27.

42. Wang X, Lu Z, Gomez A, Hon GC, Yue Y, Han D, Fu Y, Parisien M, Dai Q, Jia G, et al. N6-methyladenosine-dependent regulation of messenger RNA stability. Nature. 2014;505(7481):117-20.

43. Shi Y, Fan S, Wu M, Zuo Z, Li X, Jiang L, Shen Q, Xu P, Zeng L, Zhou Y, et al. YTHDF1 links hypoxia adaptation and non-small cell lung cancer progression. Nat Commun. 2019;10(1):4892.

44. Du H, Zhao Y, He J, Zhang Y, Xi H, Liu M, Ma J, Wu L. YTHDF2 destabilizes $\mathrm{m}(6) \mathrm{A}$-containing RNA through direct recruitment of the CCR4-NOT deadenylase complex. Nat Commun. 2016;7:12626.

45. Shi H, Wang X, Lu Z, Zhao BS, Ma H, Hsu PJ, Liu C, He C. YTHDF3 facilitates translation and decay of N(6)-methyladenosine-modified RNA. Cell Res. 2017;27(3):315-28.

46. Liu N, Zhou KI, Parisien M, Dai Q, Diatchenko L, Pan T. N6-methyladenosine alters RNA structure to regulate binding of a low-complexity protein. Nucleic Acids Res. 2017;45(10):6051-63.

47. Alarcon CR, Goodarzi H, Lee H, Liu X, Tavazoie S, Tavazoie SF. HNRNPA2B1 is a mediator of m(6)A-dependent nuclear RNA processing events. Cell. 2015;162(6):1299-308.

48. Huang H, Weng H, Sun W, Qin X, Shi H, Wu H, Zhao BS, Mesquita A, Liu C, Yuan CL, et al. Recognition of RNA N(6)-methyladenosine by IGF2BP proteins enhances mRNA stability and translation. Nat Cell Biol. 2018;20(3):285-95.

49. Wu R, Li A, Sun B, Sun JG, Zhang J, Zhang T, Chen Y, Xiao Y, Gao Y, Zhang Q, et al. A novel m(6)A reader Prrc2a controls oligodendroglial specification and myelination. Cell Res. 2019;29(1):23-41.

50. Panneerdoss S, Eedunuri VK, Yadav P, Timilsina S, Rajamanickam S, Viswanadhapalli S, Abdelfattah N, Onyeagucha BC, Cui X, Lai Z, et al. Cross-talk among writers, readers, and erasers of $\mathrm{m}(6) \mathrm{A}$ regulates cancer growth and progression. Sci Adv. 2018;4(10):eaar8263.

51. Barabasi AL, Gulbahce N, Loscalzo J. Network medicine: a network-based approach to human disease. Nat Rev Genet. 2011;12(1):56-68.

52. Lan Q, Liu PY, Haase J, Bell JL, Huttelmaier S, Liu T. The critical role of RNA m(6)A methylation in cancer. Cancer Res. 2019;79(7):1285-92.

53. Wang T, Kong S, Tao M, Ju S. The potential role of RNA N6-methyladenosine in cancer progression. Mol Cancer. 2020;19(1):88.

54. Visvanathan A, Patil V, Arora A, Hegde AS, Arivazhagan A, Santosh V, Somasundaram K. Essential role of METTL3-mediated $\mathrm{m}(6)$ A modification in glioma stem-like cells maintenance and radioresistance. Oncogene. 2018;37(4):522-33.

55. Cui Q, Shi H, Ye P, Li L, Qu Q, Sun G, Sun G, Lu Z, Huang Y, Yang CG, et al. m(6)A RNA methylation regulates the selfrenewal and tumorigenesis of glioblastoma stem cells. Cell Rep. 2017;18(11):2622-34.

56. Visvanathan A, Patil V, Abdulla S, Hoheisel JD, Somasundaram K. N(6)-methyladenosine landscape of glioma stem-like cells: 
METTL3 is essential for the expression of actively transcribed genes and sustenance of the oncogenic signaling. Genes (Basel). 2019;10(2):141.

57. Li T, Hu PS, Zuo Z, Lin JF, Li X, Wu QN, Chen ZH, Zeng ZL, Wang F, Zheng J, et al. METTL3 facilitates tumor progression via an m(6)A-IGF2BP2-dependent mechanism in colorectal carcinoma. Mol Cancer. 2019;18(1):112.

58. Deng R, Cheng Y, Ye S, Zhang J, Huang R, Li P, Liu H, Deng Q, Wu X, Lan P, et al. m(6)A methyltransferase METTL3 suppresses colorectal cancer proliferation and migration through p38/ERK pathways. Onco Targets Ther. 2019;12:4391-402.

59. Hou J, Zhang H, Liu J, Zhao Z, Wang J, Lu Z, Hu B, Zhou J, Zhao Z, Feng M, et al. YTHDF2 reduction fuels inflammation and vascular abnormalization in hepatocellular carcinoma. Mol Cancer. 2019;18(1):163.

60. Sheng H, Li Z, Su S, Sun W, Zhang X, Li L, Li J, Liu S, Lu B, Zhang S, et al. YTH domain family 2 promotes lung cancer cell growth by facilitating 6-phosphogluconate dehydrogenase mRNA translation. Carcinogenesis. 2020;41(5):541-50.

61. Paris J, Morgan M, Campos J, Spencer GJ, Shmakova A, Ivanova I, Mapperley C, Lawson H, Wotherspoon DA, Sepulveda C, et al. Targeting the RNA m(6)A reader YTHDF2 selectively compromises cancer stem cells in acute myeloid leukemia. Cell Stem Cell. 2019;25(1):137-148e136.

62. Niu Y, Lin Z, Wan A, Chen H, Liang H, Sun L, Wang Y, Li X, Xiong XF, Wei B, et al. RNA N6-methyladenosine demethylase FTO promotes breast tumor progression through inhibiting BNIP3. Mol Cancer. 2019;18(1):46.

63. Ott PA, Hu Z, Keskin DB, Shukla SA, Sun J, Bozym DJ, Zhang W, Luoma A, Giobbie-Hurder A, Peter L, et al. An immunogenic personal neoantigen vaccine for patients with melanoma. Nature. 2017;547(7662):217-21

64. Benci JL, Xu B, Qiu Y, Wu TJ, Dada H, Twyman-Saint Victor C, Cucolo L, Lee DSM, Pauken KE, Huang AC, et al. Tumor interferon signaling regulates a multigenic resistance program to immune checkpoint blockade. Cell. 2016;167(6):1540-1554 e512.

65. Zhou S, Bai ZL, Xia D, Zhao ZJ, Zhao R, Wang YY, Zhe H. FTO regulates the chemo-radiotherapy resistance of cervical squamous cell carcinoma (CSCC) by targeting beta-catenin through mRNA demethylation. Mol Carcinog. 2018;57(5):590-7.

66. Yan F, Al-Kali A, Zhang Z, Liu J, Pang J, Zhao N, He C, Litzow MR, Liu S. A dynamic N(6)-methyladenosine methylome regulates intrinsic and acquired resistance to tyrosine kinase inhibitors. Cell Res. 2018;28(11):1062-76.

67. Chen M, Nie ZY, Wen XH, Gao YH, Cao H, Zhang SF. m6A RNA methylation regulators can contribute to malignant progression and impact the prognosis of bladder cancer. Biosci Rep. 2019;39(12):BSR20192892.

68. Chen J, Yu K, Zhong G, Shen W. Identification of a m(6)A RNA methylation regulators-based signature for predicting the prognosis of clear cell renal carcinoma. Cancer Cell Int. 2020;20:157.

69. Siegel RL, Miller KD, Jemal A. Cancer statistics, 2017. CA Cancer J Clin. 2017;67(1):7-30.

70. Paulson KG, Gupta D, Kim TS, Veatch JR, Byrd DR, Bhatia S, Wojcik K, Chapuis AG, Thompson JA, Madeleine MM, et al. Agespecific incidence of melanoma in the United States. JAMA Dermatol. 2020;156(1):57-64.

71. Weber J, Mandala M, Del Vecchio M, Gogas HJ, Arance AM, Cowey CL, Dalle S, Schenker M, Chiarion-Sileni V, Marquez-Rodas I, et al. Adjuvant nivolumab versus ipilimumab in resected stage III or IV melanoma. N Engl J Med. 2017;377(19):1824-35.

72. Robert C, Schachter J, Long GV, Arance A, Grob JJ, Mortier L, Daud A, Carlino MS, McNeil C, Lotem M, et al. Pembrolizumab versus ipilimumab in advanced melanoma. N Engl J Med. 2015;372(26):2521-32.
73. Nikolaou V, Stratigos AJ. Emerging trends in the epidemiology of melanoma. Br J Dermatol. 2014;170(1):11-9.

74. Davies H, Bignell GR, Cox C, Stephens P, Edkins S, Clegg S, Teague J, Woffendin H, Garnett MJ, Bottomley W, et al. Mutations of the BRAF gene in human cancer. Nature. 2002;417(6892):949-54.

75. Flaherty KT, Yasothan U, Kirkpatrick P. Vemurafenib. Nat Rev Drug Discov. 2011;10(11):811-2.

76. Flaherty KT, Infante JR, Daud A, Gonzalez R, Kefford RF, Sosman J, Hamid O, Schuchter L, Cebon J, Ibrahim N, et al. Combined BRAF and MEK inhibition in melanoma with BRAF V600 mutations. N Engl J Med. 2012;367(18):1694-703.

77. Schadendorf D, Fisher DE, Garbe C, Gershenwald JE, Grob JJ, Halpern A, Herlyn M, Marchetti MA, McArthur G, Ribas A, et al. Melanoma. Nat Rev Dis Primers. 2015;1:15003.

78. Seynnaeve B, Lee S, Borah S, Park Y, Pappo A, Kirkwood JM, Bahrami A. Genetic and epigenetic alterations of TERT are associated with inferior outcome in adolescent and young adult patients with melanoma. Sci Rep. 2017;7:45704.

79. Huang FW, Hodis E, Xu MJ, Kryukov GV, Chin L, Garraway LA. Highly recurrent TERT promoter mutations in human melanoma. Science. 2013;339(6122):957-9.

80. Horn S, Figl A, Rachakonda PS, Fischer C, Sucker A, Gast A, Kadel S, Moll I, Nagore E, Hemminki K, et al. TERT promoter mutations in familial and sporadic melanoma. Science. 2013;339(6122):959-61.

81. Vu LP, Pickering BF, Cheng Y, Zaccara S, Nguyen D, Minuesa G, Chou T, Chow A, Saletore Y, MacKay M, et al. The N(6)-methyladenosine (m(6)A)-forming enzyme METTL3 controls myeloid differentiation of normal hematopoietic and leukemia cells. Nat Med. 2017;23(11):1369-76.

82. Dahal U, Le K, Gupta M. RNA m6A methyltransferase METTL3 regulates invasiveness of melanoma cells by matrix metallopeptidase 2. Melanoma Res. 2019;29(4):382-9.

83. Liu L, Wang Y, Wu J, Liu J, Qin Z, Fan H. N6-methyladenosine: a potential breakthrough for human cancer. Molecular Therapy Nucleic Acids. 2020;19:804-13.

84. Jia G, Fu Y, Zhao X, Dai Q, Zheng G, Yang Y, Yi C, Lindahl T, Pan T, Yang YG, et al. N6-methyladenosine in nuclear RNA is a major substrate of the obesity-associated FTO. Nat Chem Biol. 2011;7(12):885-7.

85. Yang S, Wei J, Cui YH, Park G, Shah P, Deng Y, Aplin AE, Lu Z, Hwang S, He C, et al. m(6)A mRNA demethylase FTO regulates melanoma tumorigenicity and response to anti-PD-1 blockade. Nat Commun. 2019;10(1):2782

86. Kleffel S, Posch C, Barthel SR, Mueller H, Schlapbach C, Guenova E, Elco CP, Lee N, Juneja VR, Zhan Q, et al. Melanoma cell-intrinsic PD-1 receptor functions promote tumor growth. Cell. 2015;162(6):1242-56.

87. Boussiotis VA. Molecular and biochemical aspects of the PD-1 checkpoint pathway. N Engl J Med. 2016;375(18):1767-78.

88. Jia R, Chai P, Wang S, Sun B, Xu Y, Yang Y, Ge S, Jia R, Yang YG, Fan X. m(6)A modification suppresses ocular melanoma through modulating HINT2 mRNA translation. Mol Cancer. 2019;18(1):161.

89. Li T, Gu M, Deng A, Qian C. Increased expression of YTHDF1 and HNRNPA2B1 as potent biomarkers for melanoma: a systematic analysis. Cancer Cell Int. 2020;20:239.

90. Manners O, Baquero-Perez B, Whitehouse A. m(6)A: Widespread regulatory control in virus replication. Biochim Biophys Acta Gene Regul Mech. 2019;1862(3):370-81.

91. Xue M, Zhao BS, Zhang Z, Lu M, Harder O, Chen P, Lu Z, Li A, Ma $\mathrm{Y}, \mathrm{Xu}$ Y, et al. Viral N(6)-methyladenosine upregulates replication and pathogenesis of human respiratory syncytial virus. Nat Commun. 2019;10(1):4595.

92. Kmietczyk V, Riechert E, Kalinski L, Boileau E, Malovrh E, Malone B, Gorska A, Hofmann C, Varma E, Jurgensen L et al. m(6)A-mRNA 
methylation regulates cardiac gene expression and cellular growth. Life Sci Alliance. 2019: https://doi.org/10.26508/lsa.201800233

93. Chen J, Zhang YC, Huang C, Shen H, Sun B, Cheng X, Zhang YJ, Yang YG, Shu Q, Yang Y, et al. m(6)A regulates neurogenesis and neuronal development by modulating histone methyltransferase Ezh2. Genom Proteom Bioinform. 2019;17(2):154-68.

94. Taketo K, Konno M, Asai A, Koseki J, Toratani M, Satoh T, Doki Y, Mori M, Ishii H, Ogawa K. The epitranscriptome m6A writer METTL3 promotes chemo- and radioresistance in pancreatic cancer cells. Int J Oncol. 2018;52(2):621-9.

95. Durbin AF, Wang C, Marcotrigiano J, Gehrke L: RNAs Containing Modified Nucleotides Fail To Trigger RIG-I Conformational
Changes for Innate Immune Signaling. mBio 2016: https://doi.org/ 10.1128/mBio.00833-16

96. Li HB, Tong J, Zhu S, Batista PJ, Duffy EE, Zhao J, Bailis W, Cao G, Kroehling L, Chen Y, et al. m(6)A mRNA methylation controls $\mathrm{T}$ cell homeostasis by targeting the IL-7/STAT5/SOCS pathways. Nature. 2017;548(7667):338-42.

Publisher's Note Springer Nature remains neutral with regard to jurisdictional claims in published maps and institutional affiliations. 